\title{
Performance Analysis of Parameter Estimator Based on Closed-Form Newton Method for Ultrawideband Positioning
}

\author{
Chuanyang Wang $\mathbb{D}^{1,2}$, Yipeng Ning $\mathbb{D}^{3},{ }^{3}$ Jian Wang, ${ }^{4}$ Guobiao Yao, ${ }^{3}$ Longping Zhang, ${ }^{5}$ \\ and Haichao Yang' \\ ${ }^{1}$ NASG Key Laboratory for Land Environment and Disaster Monitoring, China University of Mining and Technology (CUMT), \\ Xuzhou 221116, China \\ ${ }^{2}$ School of Environment Science and Spatial Informatics, China University of Mining and Technology (CUMT), \\ Xuzhou 221116, China \\ ${ }^{3}$ School of Surveying and Geo-Informatics, Shandong Jianzhu University, Jinan 250101, China \\ ${ }^{4}$ School of Geomatics and Urban Information, Beijing University of Civil Engineering and Architecture (BUCEA), \\ Beijing 100044, China \\ ${ }^{5}$ Piesat Information Technology Co., Ltd., Beijing 100195, China \\ ${ }^{6}$ CUMT Geotechnical Engineering \& New Technology Development Co., Ltd., Xuzhou 221116, China
}

Correspondence should be addressed to Yipeng Ning; ningyipeng19@sdjzu.edu.cn

Received 27 April 2020; Accepted 8 July 2020; Published 17 August 2020

Academic Editor: Neale R. Smith

Copyright (C) 2020 Chuanyang Wang et al. This is an open access article distributed under the Creative Commons Attribution License, which permits unrestricted use, distribution, and reproduction in any medium, provided the original work is properly cited.

\begin{abstract}
Ultrawideband (UWB) is considered as a promising technology in indoor positioning due to its accurate time resolution and good penetration through objects. Since the functional model of UWB positioning is nonlinear, iterative algorithms are often considered for solving the nonlinear problem. With a rough initial value, we can obtain the optimal solution by the way of continuous iteration. As an iterative descent method of high efficiency, the Gauss-Newton method is widely used to estimate the position. However, in the UWB indoor positioning, since the location information of the user is rather limited, it is not easy to get a good initial value for iteration. Besides, the positioning system is prone to become ill-posed. These factors make the Gauss-Newton method not easy to converge to a global optimal solution or even diverge, especially under ill-conditioned positioning configuration. Furthermore, the linearization of the positioning functional model results in biased least-squares estimation. The Gauss-Newton method only includes the first-order Taylor expansion of distance equations. The bias comes from neglected higher order terms. In this study, the closed-form Newton method which considers the high order partial derivatives is introduced and proposed. The simulation and measurement experiments are implemented to analyze the performance of the closed-form Newton method in UWB positioning. Both the initial value factor and geometric configuration factor are discussed. Experimental results are given to demonstrate that the closed-form Newton method can converge to the global optimal solution with better convergence and higher efficiency regardless of whether the initial iteration value is reasonable or not. Meanwhile, the closed-form Newton method can improve the accuracy of positioning results, particularly when the anchors are not uniformly and symmetrically distributed, or the ranging error is relatively large. The study shows that closed-form of the Newton method has better convergence and positioning performance than the Gauss-Newton method, especially under ill-conditioned positioning configuration or relatively low measurement precision, even though it adds a little computational cost.
\end{abstract}

\section{Introduction}

During recent decades, with the development of global navigation satellite system (GNSS), people pay more and more attention to the location based on service [1]. Urban dwellers spend more than $80 \%$ of their time indoors; however, the GNSS signal is not sufficiently strong to penetrate through different materials used in construction, and the phenomena of reflection and multipath fading limit the utility of GNSS in dense urban or in the indoor 
environments, which makes the indoor positioning demand growing [2]. In general, indoor localization technologies include Bluetooth, Wi-Fi, radio-frequency identification (RFID), and ultrawideband (UWB). When compared with other localization technologies, the UWB is capable of providing robust signaling, through-wall propagation and provides a large bandwidth that allows high-resolution ranging even in harsh environments [3]. Therefore, UWB technology is well suited for indoor positioning applications. In order to employ this technology, different positioning methods have been developed, such as the time-of-arrival (TOA), time difference of arrival (TDOA), angle of arrival (AOA), and received signal strength (RSS) [4].

The positioning model based on UWB observations is nonlinear. The nonlinearity in the nonlinear inversion of geodetic and geophysical data is diagnosed, the amount of nonlinearity in nonlinear models is assessed to prove whether a linearized model is a sufficient approximation, and the significance of nonlinearity is tested in the problem of nonlinear least-squares inversion [5]. The estimator is not optimum with solving the linear equations directly via least squares [6]. The polynomial approaches solve directly the nonlinear distance equations for the coordinates of the unknown station and eliminates variables appearing in the nonlinear distance equations leaving univariate polynomials [7]. The Gauss-Jacobi combinatorial procedure uses the algebraic procedures of multipolynomial resultant or Groebner basis to solve the equations contained in the minimum combinatorial sets to give closed-form solutions [8]. Although the algebraic techniques solve distance nonlinear system of equations without linearization, iteration, and forward and backward substitution, they do not solve all general types of nonlinear equations, but those nonlinear systems of equations can be converted into algebraic form because most nonlinear least-squares problem cannot be solved by algebraic methods $[9,10]$.

In general, the nonlinear algorithms can be divided into two families, noniterative and iterative. The noniterative algorithm often employs clever algebra method in order to produce a simple direct solution $[11,12]$. The most straightforward way to estimate the position may be directly solving a set of simultaneous equations [13]. However, it cannot make full use of extra measurements available to improve the accuracy. The closed-form solutions for localization using ranging difference measurements, including the spherical interpolation method, the spherical intersection method, and the plane intersection method, have been developed. To give an improved position estimate, the quadratic-correction leastsquares approach has been proposed using a two-stage weighted least-squares to solve for the source position, and the linear-correction least-squares approach has been devised with incorporating the relation explicitly by minimizing a constrained LS function $[14,15]$. The traditional positioning algorithms solve the nonlinear distance equations by linearizing them, and then, the solution can be found iteratively [16]. Iterative algorithms are the procedures that are from a rough initial guess to generate a sequence of improving approximate solutions for nonlinear equations [15]. The iterative approaches require and rely heavily on the initial starting values to ensure global minimum and faster convergence to the correct solution [17]. The numerical characteristics of a number of iterative descent algorithms for solving nonlinear least-squares problem have been discussed, particularly in the Gauss-Newton method [18]. The estimators are usually inherently biased by linearizing the nonlinear expression, and the bias comes from nonzero higher order terms [19].

A standard method for solving the nonlinear equations is Gauss-Newton iteration which is efficient and has a linear rate of convergence for points close to the solution [18]. However, the Gauss-Newton method is based on linearization and may thus perform poorly when the base stations are ill conditioned and the measurement equations are strongly nonlinear. Tikhonov regularization is the most commonly used method for nonlinear ill-posed problems; the chosen Tikhonov matrix gives preference to solutions with smaller norms which is known as L2 regularization [20]. The regularization and Gauss-Newton method are combined into the iteratively regularized Gauss-Newton method to deal with the matrix rank deficient problems [21]. The Gauss-Newton method strengthened with a regularization term is found to be as accurate as any of the closedform methods through simulation comparison, while being simpler to implement and avoiding most of the pitfalls [22]. As one kind of the steepest decent method, the barycenter method is discussed. It can stabilize the iteration without the matrix inversion and simplify the procedure without calculating the Hessian matrix [23]. The trust region and damped methods are introduced and modified for nonlinear least squares $[24,25]$. The closed-form of the Newton method by which the Newton method and Gauss-Newton method are connected has been obtained for solving the distance equations $[26,27]$. To minimize the objective function for the optimal position estimation, the performance of Gauss-Newton type and the quasi-Newton methods is analyzed and discussed [28]. To get a better approximation of the Hessian matrix of the objective function, the Broyden-Fletcher-Goldfarb-Shanno (BFGS) algorithm and Davidon-Fletcher-Powell (DFP) algorithm in the quasi-Newton methods have been compared [29].

The remainder of this paper is organized as follows. In Section 2, the nonlinear distance equations for UWB positioning are introduced. In Section 3, the closed-form Newton iterative method is presented. In Section 4, the performance of the closed-form Newton method in UWB positioning is analyzed and illustrated by the numerical examples. In Section 5, the conclusions are summarized.

\section{The Nonlinear Distance Equations for UWB Positioning}

As shown in Figure 1, we evaluate the UWB sensor network positioning system based on the DW1000 module. It allows high data rate communications, up to $6.8 \mathrm{Mb} / \mathrm{s}$, and it is a perfect fit in wireless sensor network applications [30]. By 


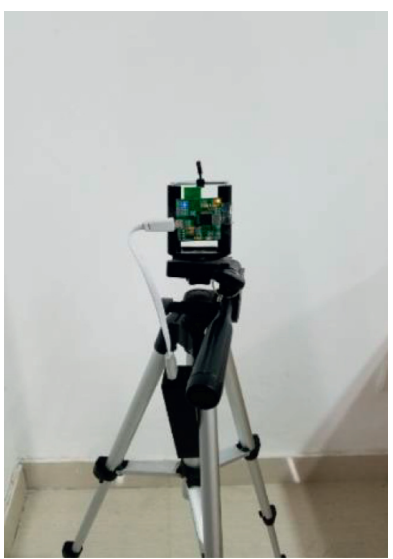

(a)

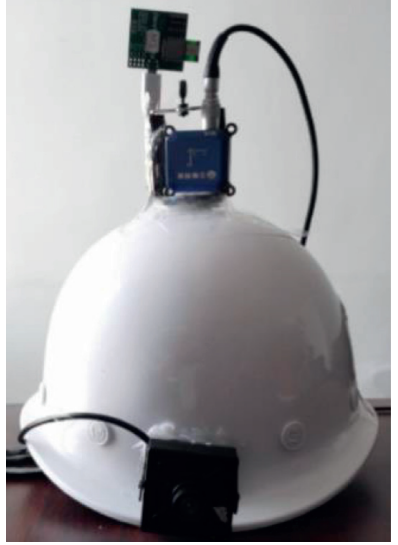

(b)
Figure 1: The anchor (a) and tag (b) hardware devices.

determining the time of flight (TOF) of signals travelling between the anchor and tag, the ranging measurements in these transceivers is performed based on the two way ranging (TWR).

After collecting ranging data by the positioning system, we use the TOA method to process the data for positioning calculation. The three-dimensional (3D) position of the tag is determined as the intersection of all the spheres, whose centers are the coordinates of the anchors and radius is the range between the anchors and the tag. However, the accuracy of ranging distance is affected by noise and the multipath components; thus, the spheres do not always intersect at one single point. The goal of the positioning estimation is to find out coordinates closest to the actual position [31].

The nonlinear observation model of distance equation is given as [32]

$$
L_{i}=d_{i}(\mathbf{X})+\varepsilon_{i} \quad(i=1,2, \ldots, n)
$$

where $L_{i}$ denotes the observation distance between the tag and $i$ th anchor; $\mathbf{L}=\left[\begin{array}{llll}L_{1} & L_{2} & \ldots & L_{n}\end{array}\right]^{\mathrm{T}}$ denotes the observation vector; $d_{i}(\mathbf{X})=\sqrt{\left(x_{i}-x\right)^{2}+\left(y_{i}-y\right)^{2}+\left(z_{i}-z\right)^{2}}$ is the Euclidean distance; $\varepsilon_{i}$ represents the corresponding random error; $\left(x_{i}, y_{i}, z_{i}\right)$ is the known coordinate of $i$ th anchor; and $(x, y, z)$ is the coordinate of tag.

The tag coordinates can be estimated by the nonlinear equations. If the nonlinear equations are overdetermined, then the nonlinear least-squares solution is to find $\widehat{\mathbf{X}}=$ $\operatorname{argmin}_{\mathbf{X}} g(\mathbf{X})$, where $g(\mathbf{X})=\mathbf{V}^{\mathbf{T}}(\mathbf{X}) \mathbf{V}(\mathbf{X}) / 2$, in which $\mathbf{V}(\mathbf{X})=\mathrm{d}(\mathbf{X})-\mathbf{L}$ represents the residual vector and $\mathbf{P}(\mathbf{X})=$ $\operatorname{diag}\left(p_{1}, p_{2}, \ldots, p_{n}\right)$ is the weight matrix. We denote $g(\mathbf{X})$ as the least-squares objective function, since the function is twice continuously differentiable; then, its first-order partial derivatives are obtained:

$$
\mathbf{h}(\mathbf{X})=g^{\prime}(\mathbf{X})=\mathbf{J}^{\mathrm{T}}(\mathbf{X}) \mathbf{P V}(\mathbf{X}),
$$

where $\mathbf{J}(\mathbf{X})$ represents the Jacobian matrix:

$$
\mathbf{J}(\mathbf{X})=\left[\begin{array}{c}
\mathbf{e}_{1} \\
\mathbf{e}_{2} \\
\vdots \\
\mathbf{e}_{n}
\end{array}\right]=\left[\begin{array}{ccc}
e_{11} & e_{12} & e_{13} \\
e_{21} & e_{22} & e_{23} \\
\vdots & \vdots & \vdots \\
e_{n 1} & e_{n 2} & e_{n 3}
\end{array}\right]
$$

In which $\mathbf{e}_{i}(\mathbf{X})=\left[x-x_{i}, y-y_{i}, z-z_{i}\right] / d_{i}(\mathbf{X})$ is the $i$ th direction cosine vector from the unknown point to the $i$ th known point. The solution of the objective function can be then obtained by solving the orthogonal condition as [8]

$$
\mathbf{h}(\widehat{\mathbf{X}})=g^{\prime}(\widehat{\mathbf{X}})=\mathbf{J}^{\mathrm{T}}(\widehat{\mathbf{X}}) \mathbf{P V}(\widehat{\mathbf{X}})=0 .
$$

The nonlinear equation (4) is a base to discuss the properties of the nonlinear least-squares solution and develop iterative methods and even algebraic approaches for solving the overdetermined distance equations [26]. The nonlinearity measures may be obtained by the Hessian matrix of the form

$$
\begin{aligned}
\mathbf{H}(\widehat{\mathbf{X}}) & =\mathbf{h}^{\prime}(\widehat{\mathbf{X}}) g \prime \prime(\widehat{\mathbf{X}})=\mathbf{J}^{\mathrm{T}}(\widehat{\mathbf{X}}) \mathbf{P J}(\widehat{\mathbf{X}})+\sum_{i=1}^{n} V_{i}(\widehat{\mathbf{X}}) p_{i} V_{i}^{\prime \prime}(\widehat{\mathbf{X}}) \\
& =\mathbf{N}(\widehat{\mathbf{X}})+\mathbf{S}(\widehat{\mathbf{X}})
\end{aligned}
$$

where $V_{i}^{\prime \prime}(\widehat{\mathbf{X}})$ contains the second derivatives of the distance equation [18].

\section{The Closed-Form Newton Method}

For small residual and low nonlinearity problems in nonlinear adjustment models, a linearized model is a sufficient approximation, $\sum_{i=1}^{n} V_{i}(\mathbf{X}) p_{i} V_{i}^{\prime \prime}(\mathbf{X}) \longrightarrow 0$, the Hessian matrix $\mathbf{H}(\mathbf{X})$ can be approximated to its linear term $\mathbf{N}(\mathbf{X})$, we have the Gauss-Newton method as

$$
\mathbf{X}_{k+1}=\mathbf{X}_{k}+\mathbf{N}^{-1}\left(\mathbf{X}_{k}\right) \mathbf{h}\left(\mathbf{X}_{k}\right) \text {. }
$$

However, the issues come in when the approximation is not adequate; if $\mathbf{H}(\mathbf{X})$ is ill conditioned, $\mathbf{N}(\mathbf{X})$ will not be a sufficient approximation to the Hessian matrix, and the least-squares solution will be unstable. Moreover, the linearization of the positioning functional model results in biased least-squares estimators. The Gauss-Newton method only includes the first-order Taylor expansion of distance equations. The bias comes from neglected higher order terms, which can be regarded as a systematic error.

To stabilize the ill-conditioned least-squares solution, the Gauss-Newton method can be corrected by using prior information; this kind of prior assumption can easily be incorporated in the Gauss-Newton method as a regularization term. For the indoor applications, the preliminary information about the user position is limited, the ill-posed problems will occur when the anchors are configured close to coplanar and the measurement errors are large.

To solve the ill-conditioned configurations problems, a new iterative descent method called the closed-form Newton method is introduced [27]. The Hessian matrix can be derived from $\mathbf{h}(\mathbf{X})$, if $i=j$, then 


$$
\begin{aligned}
H_{i j}(\mathbf{X})=\partial h_{i}(\mathbf{X}) / \partial X_{i}=\partial h_{j}(\mathbf{X}) / \partial X_{j} & =\sum_{k=1}^{n} p_{k}+\sum_{k=1}^{n}\left[\frac{-p_{k} L_{k}}{d_{k}(\mathbf{X})}+\frac{-L_{k}\left(X_{j}-X_{k, j}\right)}{-d_{k}^{2}(\mathbf{X})} \frac{\partial d_{k}(\mathbf{X})}{\partial X_{j}}\right] \\
& =\sum_{k=1}^{n}\left[\frac{p_{k}\left(d_{k}(\mathbf{X})-L_{k}\right)}{d_{k}(\mathbf{X})}+\frac{p_{k} L_{k}}{d_{k}(\mathbf{X})} \frac{\left(X_{j}-X_{k, j}\right)^{2}}{d_{k}^{2}(\mathbf{X})}\right] .
\end{aligned}
$$

Similarly, we can acquire other entries, and all entries of the Hessian matrix can be presented as

$$
H_{i j}(\mathbf{X})=\partial h_{i}(\mathbf{X}) / \partial X_{j}=\left\{\begin{array}{l}
\sum_{k=1}^{n}\left[\frac{p_{k}\left(d_{k}(\mathbf{X})-L_{k}\right)}{d_{k}(\mathbf{X})}+\frac{p_{k} L_{k}}{d_{k}(\mathbf{X})} \frac{\left(X_{j}-X_{k, j}\right)^{2}}{d_{k}^{2}(\mathbf{X})}\right] \quad i=j, \\
\sum_{k=1}^{n}\left[\frac{p_{k} L_{k}\left(X_{i}-X_{k, i}\right)\left(X_{j}-X_{k, j}\right)}{d_{k}^{2}(\mathbf{X})}\right] \quad i \neq j .
\end{array}\right.
$$

Equation (8) is expressed as the matrix form

$$
\mathbf{H}(\mathbf{X})=\mathbf{J}^{\mathrm{T}}(\widehat{\mathbf{X}}) \mathbf{R} \mathbf{J}(\mathbf{X})+\alpha \mathbf{I},
$$

where $\operatorname{tr}(\cdot)$ is the trace of matrix, $\alpha=\operatorname{tr}(\mathbf{P})-\operatorname{tr}(\mathbf{P R})$ is the trace of matrix, and $\mathbf{R}=\operatorname{diag}\left(L_{1} / d_{1}(\mathbf{X}), L_{2} / d_{2}(\mathbf{X}), \ldots\right.$, $\left.L_{n} / d_{n}(\mathbf{X})\right)$.

Closed-form Newton iterative formula can be given as

$$
\mathbf{X}_{k+1}=\mathbf{X}_{k}+\left(\mathbf{J}^{\mathrm{T}}(\mathbf{X}) \mathbf{R} \mathbf{J}(\mathbf{X})+\alpha \mathbf{I}\right)^{-1} \mathbf{J}^{\mathrm{T}}\left(\mathbf{X}_{k}\right) \mathbf{P V}\left(\mathbf{X}_{k}\right)
$$

If the residual is small enough, $\mathbf{R} \longrightarrow \mathbf{I}$, closed-form Newton will be reduced to the Gauss-Newton method.

\section{Numerical Examples}

In this section, the simulation and measurement experiments in different situations are implemented. The iterative convergence and position estimation based on the closedform Newton method are analyzed and compared.

4.1. Simulation Verification. The positioning configurations and coordinates of the anchor and tag are shown in Figure 2. As shown in Table 1, four sets of tests are conducted in the simulation experiment. The simulated measurement errors are expressed by the standard deviation (STD). They obey the normal distribution which is an independent Gaussian variable with zero mean and equal variance. The observation model equation is simulated 10,000 times for evaluating the performance of the closed-form Newton method.

The position dilution of precision (PDOP), horizontal dilution of precision (HDOP), and vertical dilution of precision (VDOP) of positioning configurations are illustrated in Figure 3. Dilution of precision (DOP) describes the relationship between measure error and position determination. The more observations used, the smaller the DOP values, and hence the smaller the solution error. It can be seen that three types of DOP values of Configuration 1 are smaller than that of Configuration 2. In Configuration 1, there are adequate redundant observations, and the anchors are uniformly and symmetrically distributed in the threedimensional (3D) space. In Configuration 2, there is only one redundant observation with anchors distributed on a planar ground. This makes the number of parameters to be estimated relatively more for the particular observation model, and the nonlinear strength increases with the decreases of degree of freedom (DOF).

The statistical results of iterations and convergence property are shown in Figure 4 and Table 2, respectively. In Test 1, we can see that both the Gauss-Newton method and closed-form Newton method can converge to the optimal solutions with very few iterations. It indicates that these two iterative methods can converge to the global optimal solution with better convergence and higher efficiency under well-conditioned positioning configuration. Compared with Test 1 , Test 2 is based on ill-conditioned positioning configuration. Test 1 and Test 2 have the same measurement error, and meanwhile, the initial iteration values for Test 1 and Test 2 are true positions. In Test 2, Gauss Newton method diverges in some epochs. However, closed-form Newton method guarantees the convergence in all epochs although there are more iterations in some epochs. Compared with Test 2, the observation in Test 3 is more accurate, and the error is smaller. It makes known that both the both Gauss-Newton method and closed-form Newton method can achieve correct convergence if the observation accuracy is high enough despite the positioning configuration is ill conditioned. The initial iteration value for Test 4 is different from Test 2. It can be known that the initial value affects the iterations, but does not affect the convergence success rate.

The frequency histograms of positioning error based on the Gauss-Newton method closed-form Newton method are represented in Figures 5 and 6, respectively. The average positioning error under convergence is given in Table 3 . As we can see, the positioning error distribution of the Gauss-Newton 


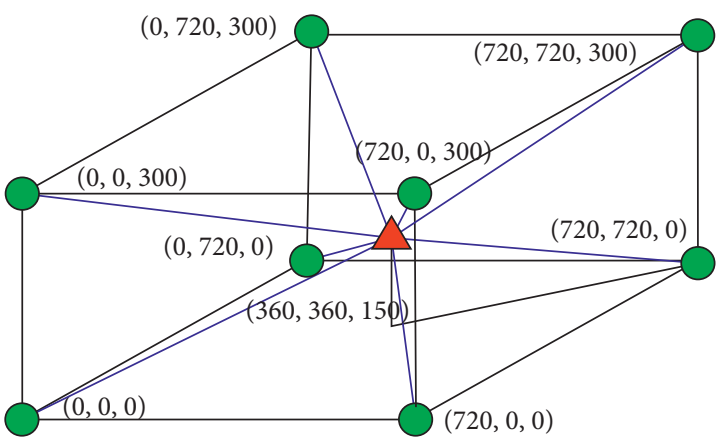

(a)

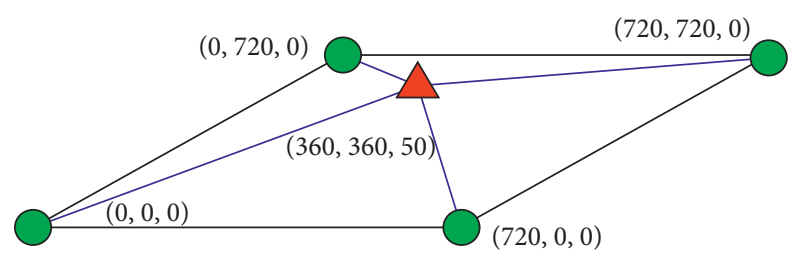

(b)

FIgURE 2: The positioning configurations of tests. (a) Configuration 1 (Test 1). (b) Configuration 2 (Tests 2, 3, and 4).

TABLe 1: The experimental setup $(\mathrm{cm})$.

\begin{tabular}{lcccc}
\hline The experimental variables & Test 1 & Test 2 & Test 3 & Test 4 \\
\hline The positioning configuration & Configuration 1 & Configuration 2 & Configuration 2 & Configuration 2 \\
The STD of observation & 3 & 3 & 1 & 3 \\
The initial value for iteration & $(360,360,150)$ & $(360,360,50)$ & $(360,360,50)$ & $(360,360,10)$ \\
\hline
\end{tabular}

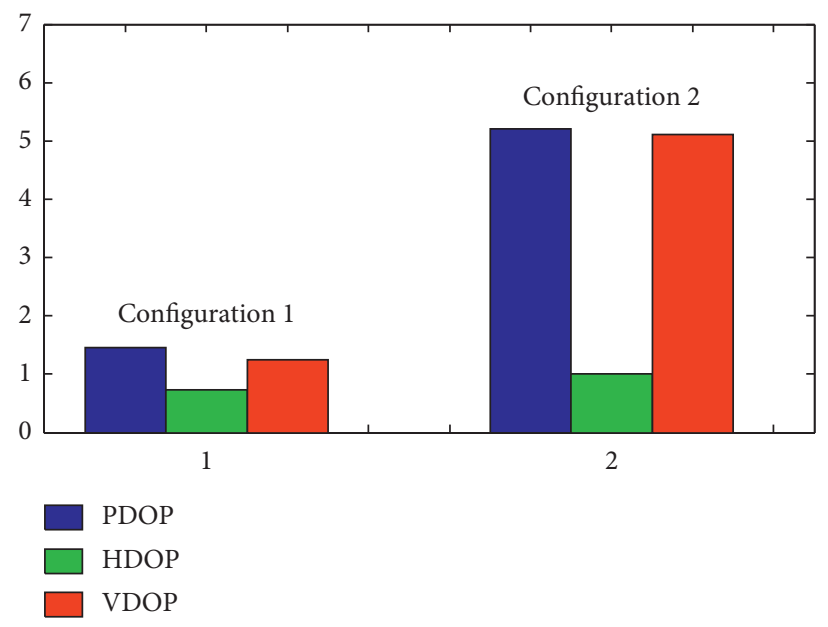

Figure 3: The PDOP, HDOP, and VDOP of positioning configurations.
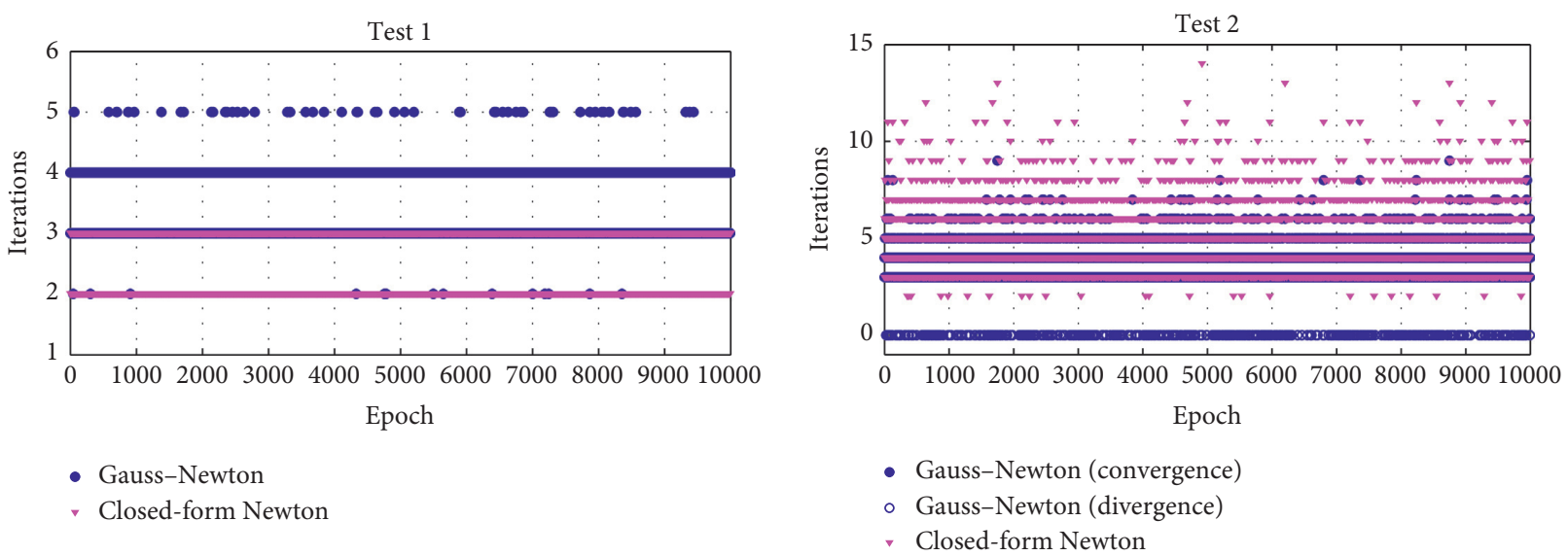

(a)

(b)

Figure 4: Continued. 


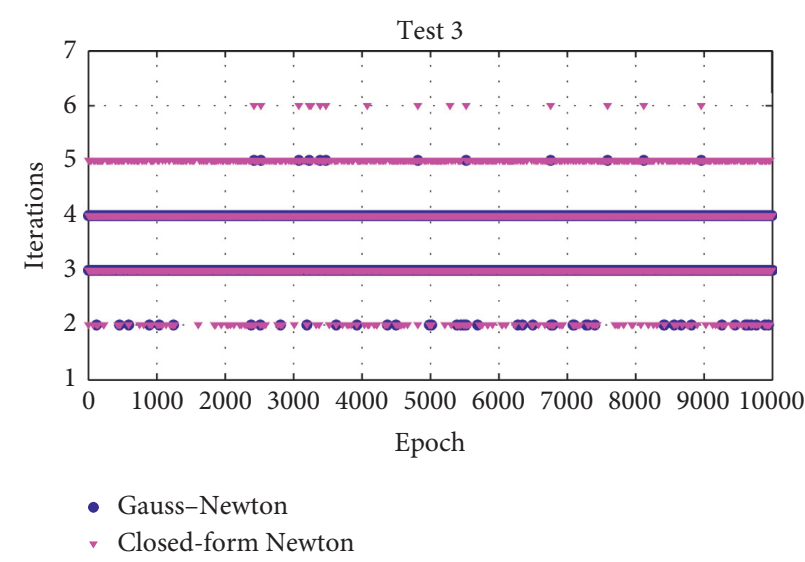

(c)

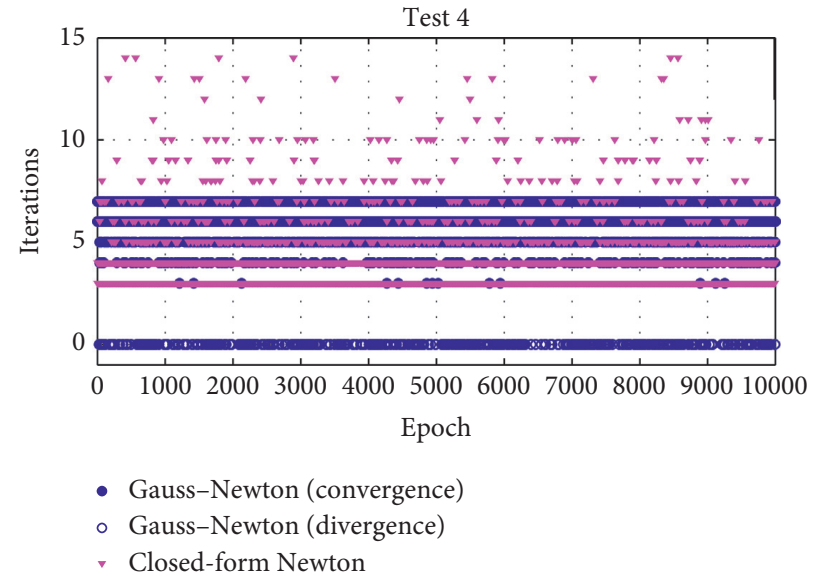

(d)

Figure 4: The statistical results of iterations. (a) Test 1 (Configuration 1). (b) Test 2 (Configuration 2). (c) Test 3 (Configuration 2 ). (d) Test 4 (Configuration 2).

TABLE 2: The statistical results of convergence property.

\begin{tabular}{lcccrr}
\hline Method & Statistics & Test 1 & Test 2 & Test 3 & Test 4 \\
\hline \multirow{2}{*}{ Gauss-Newton } & Convergence rate & 1.000 & 0.950 & 1.000 & 0.950 \\
\multirow{2}{*}{ Closed-form Newton } & Average iterations & 3.370 & 3.849 & 3.263 & 6.179 \\
& Convergence rate & 1.000 & 1.000 & 1.000 & 1.000 \\
& Average iterations & 2.350 & 4.807 & 3.676 & 3.568 \\
\hline
\end{tabular}

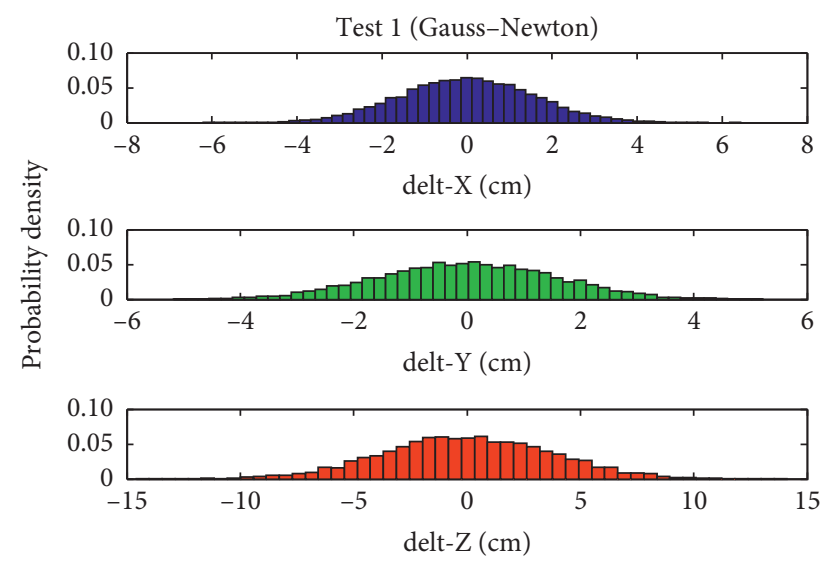

(a)

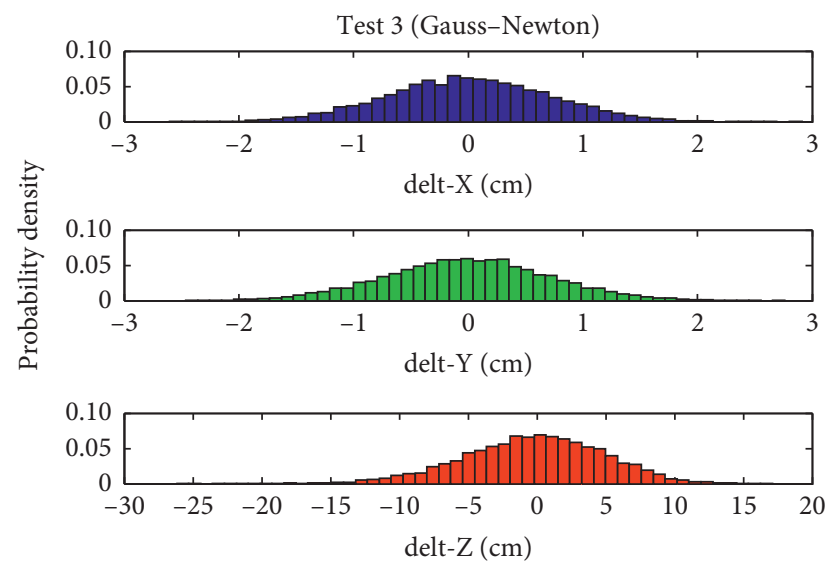

(c)

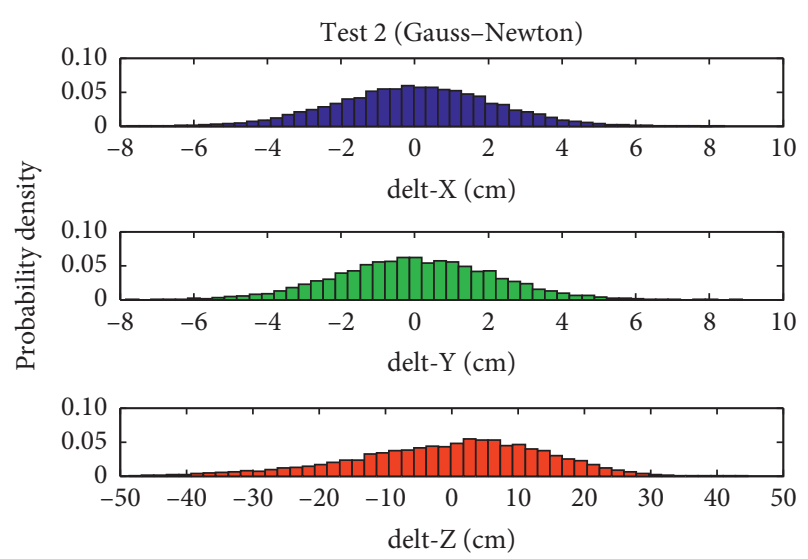

(b)
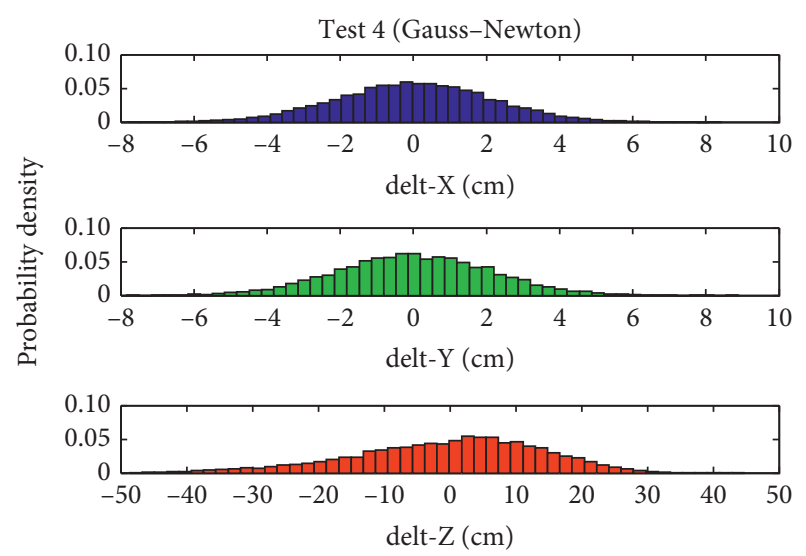

(d)

Figure 5: The frequency histograms of positioning error based on the Gauss-Newton method. (a) Test 1. (b) Test 2. (c) Test 3. (d) Test 4. 


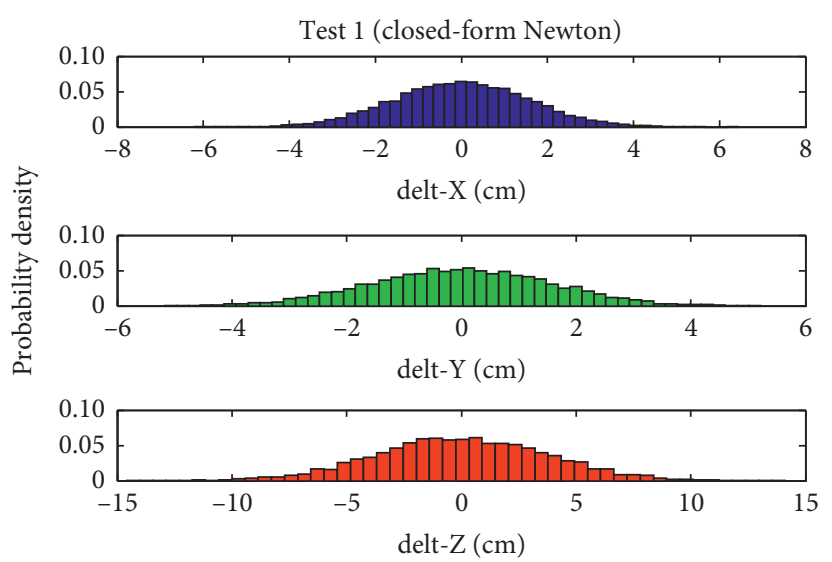

(a)

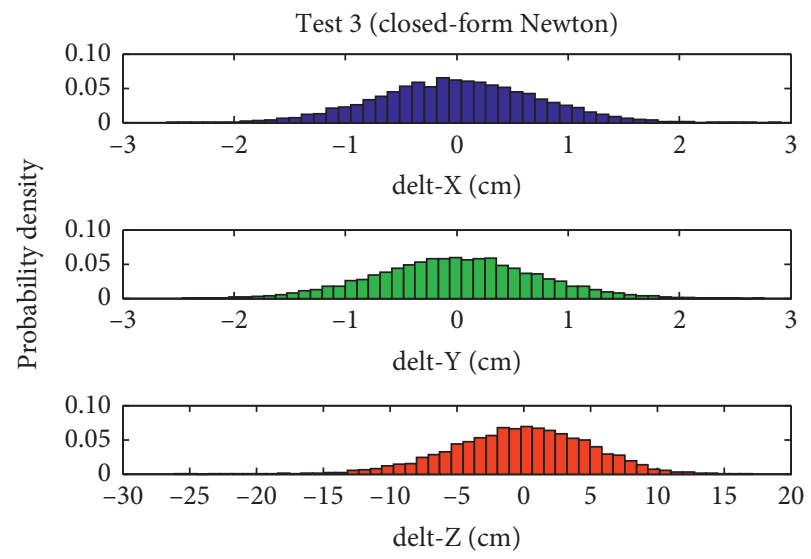

(c)

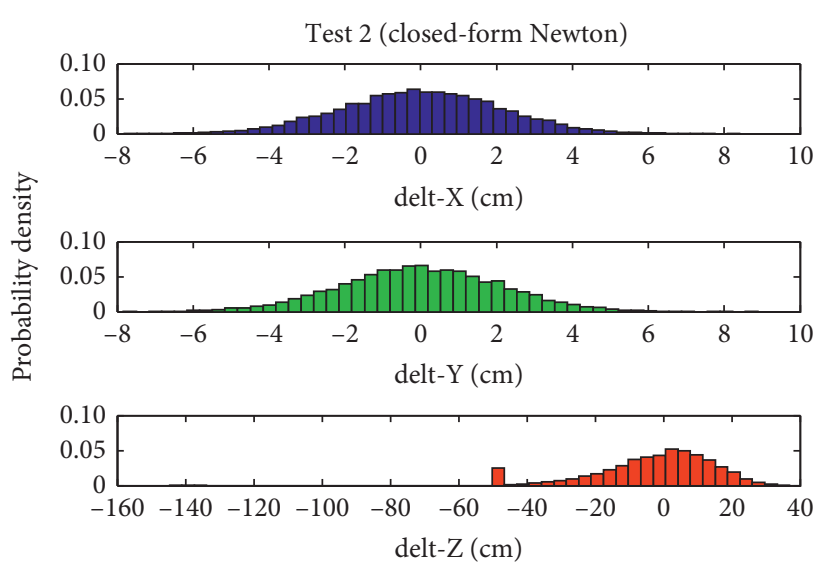

(b)

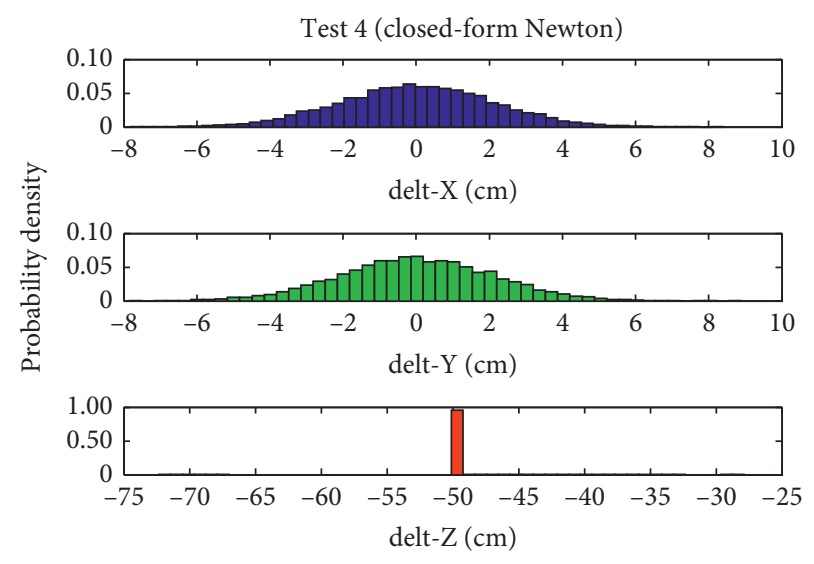

(d)

Figure 6: The frequency histograms of positioning error based on the closed-form Newton method. (a) Test 1. (b) Test 2. (c) Test 3. (d) Test 4.

TABLE 3: The average positioning error under convergence $\left(10^{-3}\right.$ $\mathrm{cm})$.

\begin{tabular}{lccccc}
\hline Method & Coordinate & Test 1 & Test 2 & Test3 & Test 4 \\
\hline \multirow{4}{*}{ Gauss-Newton } & $X$ & -13 & 16 & 2 & 16 \\
& $Y$ & -6 & 9 & -7 & 9 \\
Closed-form Newton & $Z$ & 23 & -502 & -319 & -502 \\
& $X$ & -13 & 8 & 2 & 8 \\
& $Y$ & -6 & 4 & -7 & 4 \\
& $Z$ & 23 & 38.0 & -319 & -49954 \\
\hline
\end{tabular}

method closed-form Newton method is basically the same in Test 1 and Test 3. It can be known that the performance of closed-form Newton method and Gauss-Newton method is consistent if the system geometry is well conditioned or the observation accuracy is high enough. In Test 2, the closed-form Newton method has smaller average positioning error than the Gauss-Newton method, especially in the coordinate component Z. It indicates that the closed-form Newton method has higher positioning accuracy. In Test 4, the closed-form Newton method achieves effective convergence and converge to optimal solution in the coordinate component $X$ and $Y$, but does not converge to optimal solution in the coordinate component $Z$ which is ill-conditioned direction. In practical applications, since the location information of the user is rather limited, it is not easy to get a good initial value. It can be known that the closed-form Newton method can guarantee the complete convergence by sacrificing the positioning accuracy in the illconditioned direction.

4.2. Measurement Verification. In the measurement experiment, the hardware devices used are the DecaWave Mini2016 suite, including the anchor and tag nodes, already described in detail in Section 2. The experimental scene is an empty hall. During the testing process, no relevant personnel entered. The tag node is mounted on the top of the helmet, which is placed at test point at the height of $30 \mathrm{~cm}$. The anchor nodes are placed on a tripod of the same antenna height. The main goal is to evaluate the performance of the closed-form Newton method in a static situation. The locations of test points and anchors and the experimental scene are shown in Figure 7. The experimental setup is shown in Table 4 . The positions of test points were calibrated ahead of time. In the test point, 280 epochs of data were sampled. In the following four sets of tests, there is only one redundant observation. This makes the number of parameters to be estimated relatively more for the particular observation model, and the nonlinear strength increases with the decreases of DOF. 


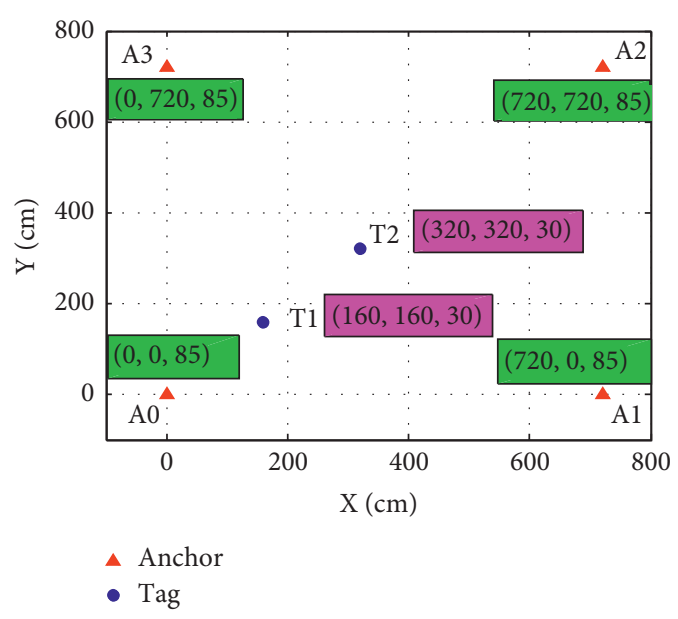

(a)

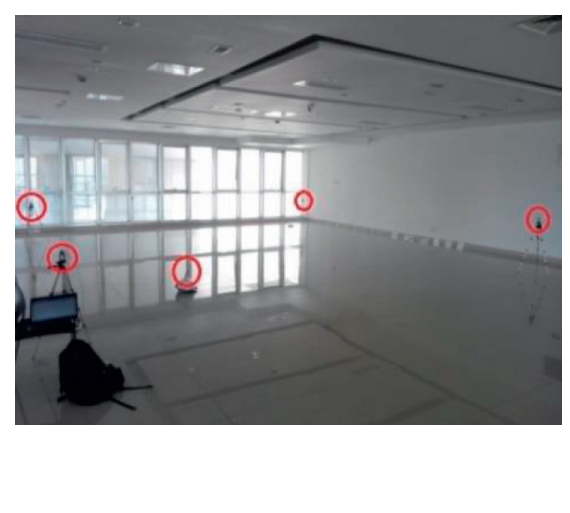

(b)

Figure 7: The locations of test points and anchors (a) and the experimental scene (b).

TABLe 4: The experimental setup $(\mathrm{cm})$.

\begin{tabular}{lcccc}
\hline The experimental variables & Test 1 & Test 2 & Test 3 & Test 4 \\
\hline Test point & T1 & T1 & T1 & T2 \\
Initial value for iteration & $(160,160,83)$ & $(160,160,70)$ & $(160,160,30)$ & $(320,320,30)$ \\
\hline
\end{tabular}

Figure 8 represents the cumulative distribution function (CDF) of the ranging error of all the measurements collected. It can be observed that the random error of these observations are small and differ slightly. However, all of their systematic errors are very large. The distance between $\mathrm{T} 1$ and A1 perfectly matches the distance between $\mathrm{T} 1$ and A3, but their range estimation accuracy is different due to the hardware. Although the hardware devices adopt the principle of TWR without a common time reference, the clock drift and offset still affect the ranging error. We can also see that the systematic error based on anchors A1 and A3 becomes larger than that based on anchors A0 and A2.

The statistical results of iterations and convergence property are shown in Figure 9 and Table 5, respectively. In Test 1 , the initial value for iteration is close to coplanar with the anchors. It can be observed that the convergence rate of the Gauss-Newton method is lowest. Meanwhile, all of the successful iterations are false convergence with the local optimal solution. Compared with Test 1 , Test 2 is based on a relatively reasonable initial value. The Gauss-Newton method converges correctly, and its convergence rate is improved obviously. Comparatively speaking, the closedform Newton method can converge quickly in all epochs. It can be known that the initial value has a great influence on the iteration of the Gauss-Newton method under the illconditioned positioning configuration. Moreover, the closed-form Newton method can converge to the global optimal solution with better convergence and higher efficiency regardless of whether the initial iteration value is reasonable or not.

In addition, the location estimation results based on Test 3 and Test 4 are expressed in Figures 10 and 11. The initial values for iteration for Test 3 and Test 4 are true positions. In Test 3, although the Gauss-Newton method converges to the global optimal solution, the positioning error in the coordinate component $Z$ is very large. The closed-form Newton method imposes constraints on the positioning in the coordinate component $Z$, and uses the $Z$-axis coordinates of the base station plane as its positioning result, which limits the $Z$-axis positioning error under the ill-conditioned configuration. This also results in more iterations of the closed-form Newton method in Test 3 than Tests 1 and 2. Furthermore, the accuracy of horizontal location estimation results of the closed-form Newton method is effectively improved compared to the Gauss-Newton method. The reason is that the Gauss-Newton method only includes the first-order Taylor expansion of distance equations due to the linearization of the positioning functional model. The bias comes from neglected higher order terms, which can be regarded as a systematic error. The closed-form Newton method which considers the high order partial derivatives is more stable and effective. It can converge to the global optimal solution with increasing the computational cost a little. In Test 4, the horizontal location estimation results of the closed-form Newton method and Gauss-Newton method are similar. The main cause for this case is that the positioning configuration of Test 4 is more symmetrical than that of Test 3 in the coordinate components $X$ and Y. The better the system geometry is, the smaller the bias in the parameter estimator will be. For a positioning configuration, when the anchors are evenly distributed, the amount of nonlinearity is orthogonal to the ranging space of the design matrix, the linearization is valid, and the parameter estimator tends to be unbiased. 

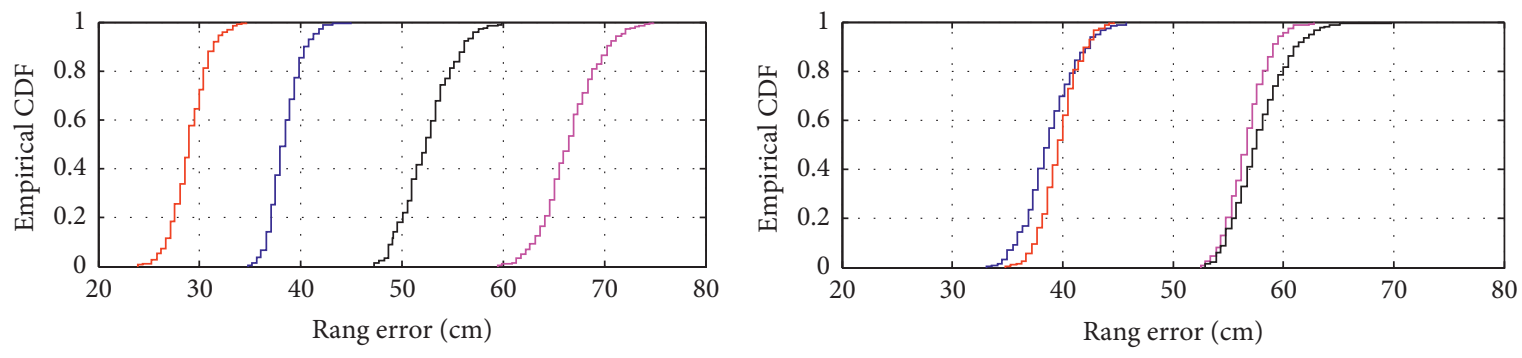

$\begin{array}{ll}\mathrm{A} 0-\mathrm{T} 1 & -\mathrm{A} 2-\mathrm{T} 1 \\ \mathrm{~A} 1-\mathrm{T} 1 & \mathrm{~A} 3-\mathrm{T} 1\end{array}$

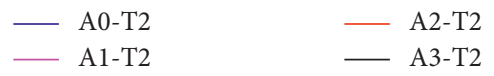

(a)

(b)

FIgURE 8: The cumulative distribution function (CDF) of the ranging error. (a) T1. (b) T2.
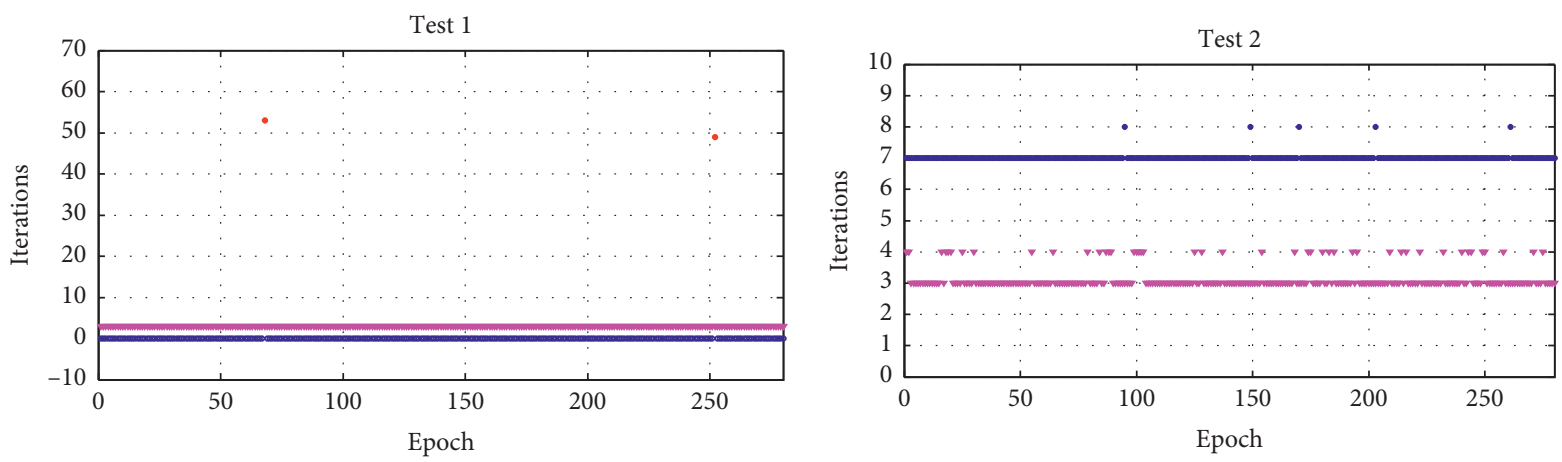

- Gauss-Newton (false convergence)

- Gauss-Newton (divergence)

v Closed-form Newton

- Gauss-Newton

- Closed-form Newton

(a)

(b)
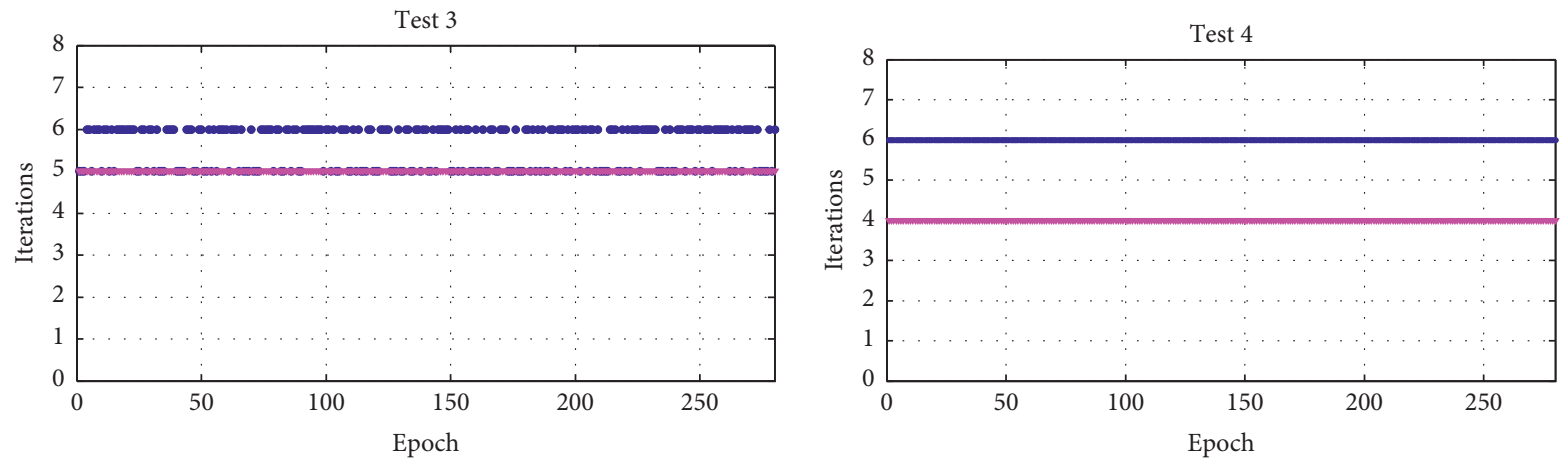

- Gauss-Newton

- Closed-form Newton

- Gauss-Newton

- Closed-form Newton

(c)

(d)

Figure 9: The statistical results of iterations. (a) Test 1. (b) Test 2. (c) Test 3. (d) Test 4.

TABle 5: The statistical results of convergence property.

\begin{tabular}{lcccrr}
\hline Methods & Statistics & Test 1 & Test 2 & Test 3 & Test 4 \\
\hline \multirow{2}{*}{ Gauss-Newton } & Convergence rate & 0.007 & 1.000 & 1.000 & 1.000 \\
\multirow{2}{*}{ Closed-form Newton } & Average iterations & 51.000 & 7.018 & 5.575 & 6.180 \\
& Convergence rate & 1.000 & 1.000 & 1.000 & 1.000 \\
& Average iterations & 3.000 & 3.161 & 5.000 & 4.000 \\
\hline
\end{tabular}



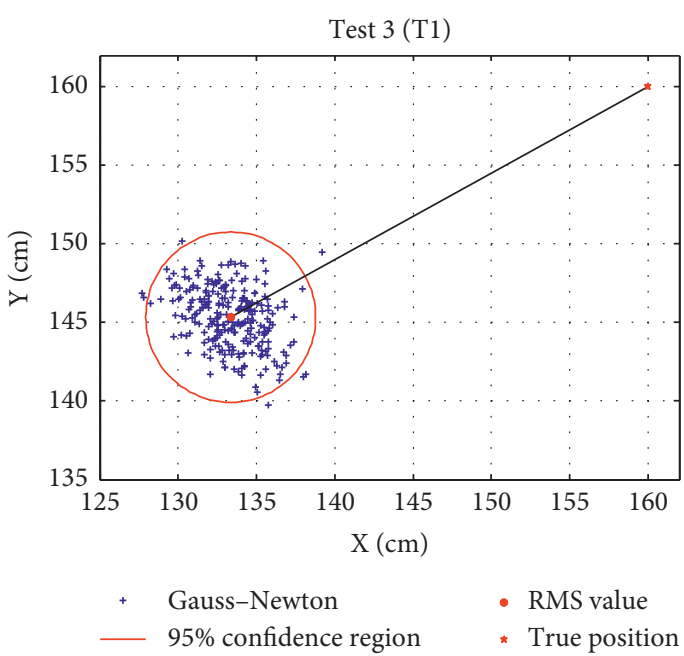

(a)

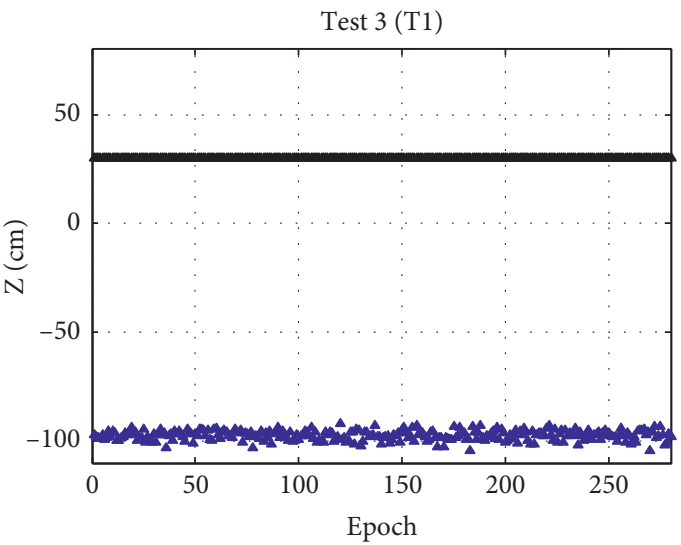

- Parameter estimator based on Gauss-Newton - True position

(c)
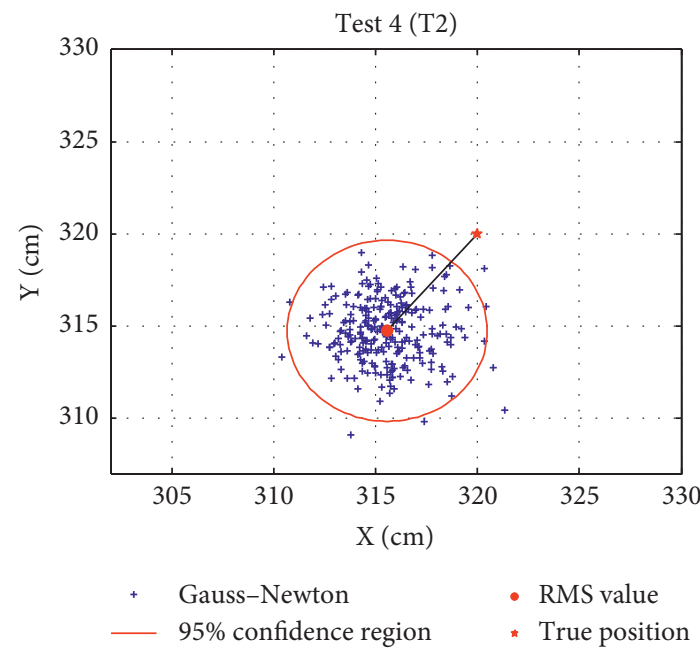

(b)

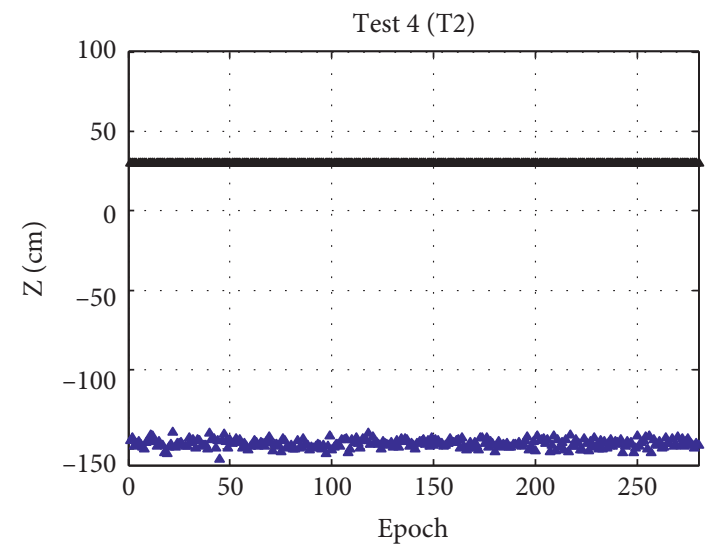

ـ Parameter estimator based on Gauss-Newton

\ True position

(d)

Figure 10: The location estimation results of the Gauss-Newton method based on Test 3 and Test 4 .

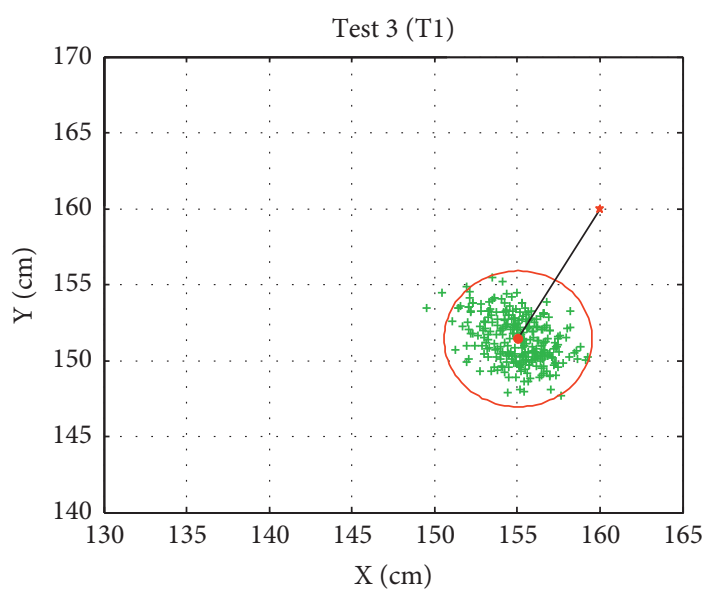

+ Closed-form Newton

- RMS value

— $95 \%$ confidence region

(a)

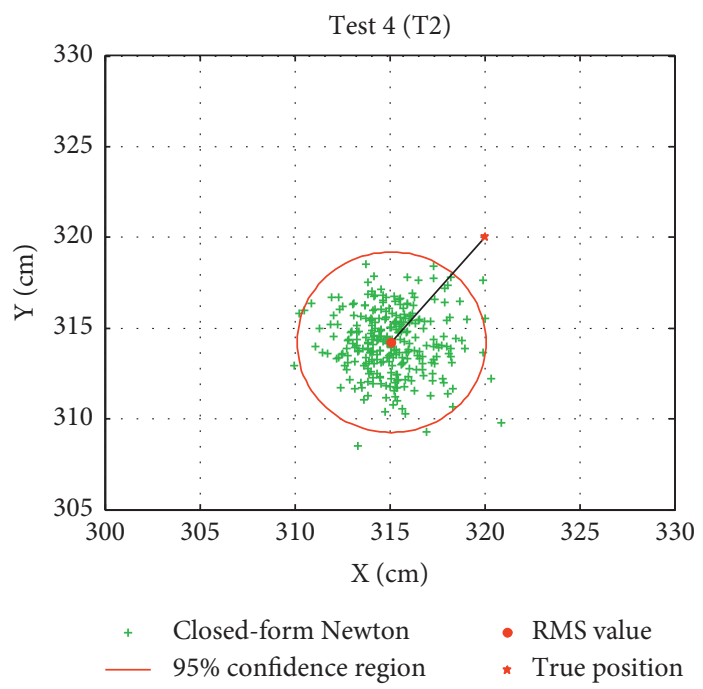

(b)

Figure 11: Continued. 


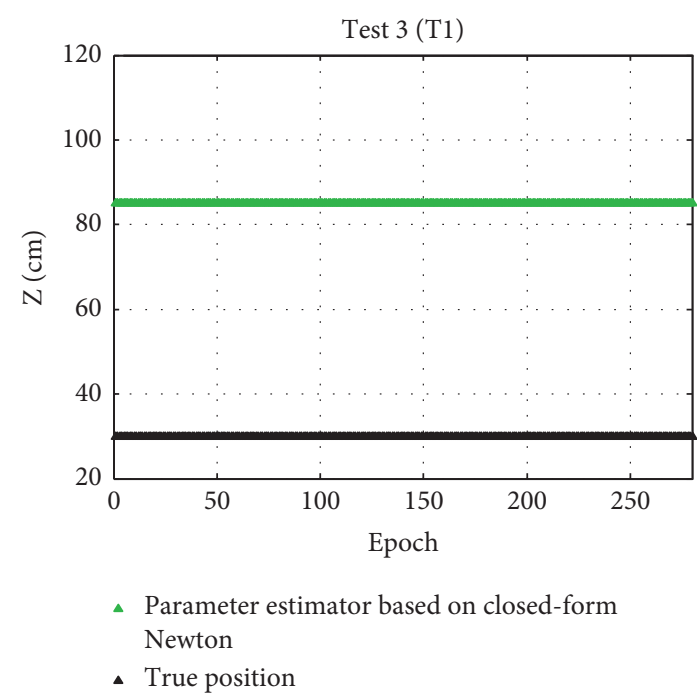

(c)

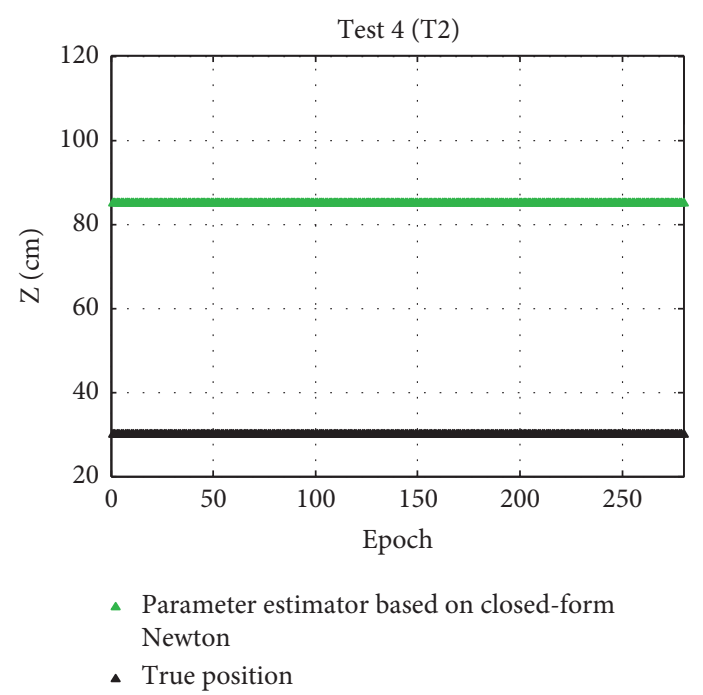

(d)

Figure 11: The location estimation results of the closed-form Newton method based on Test 3 and Test 4 .

According to the above analysis and research, we can believe that the closed-form Newton method has better convergence and higher efficiency. It confirms that the closed-form Newton method performs better than the Gauss-Newton method in ultrawideband positioning, especially under ill-conditioned configuration.

\section{Conclusions}

In this paper, we introduce the closed-form Newton method for UWB positioning. It provides an overview of the reliability of the closed-form Newton method applied to UWB positioning. The linearization of the positioning functional model results in biased least-squares estimators. The Gauss-Newton method only includes the firstorder Taylor expansion of distance equations so that it has higher computational efficiency. The bias comes from neglected higher order terms, which can be regarded as a systematic error. Besides, in outdoor satellite positioning, a good initial value can be obtained and a good positioning geometry configuration can be chosen due to many dynamic available satellites. Then, the convergence property is efficient. However, in the UWB indoor positioning, since the prior information of the user position is rather limited, it is not easy to get a good initial value. Moreover, the nonline-of-sight (NLOS) environment makes the error of measurements increased and biased. In addition, in view of the complexity and cost of the positioning system, the geometry configuration of anchor nodes is prone to be ill conditioned. When selecting an unreasonable initial value, the Gauss-Newton method converges with many iterations or diverges sometimes or converges to a false local optimal solution. The effect of nonlinearity on the positioning system can become rather straightforward. Thus, the performance and the reliability of the positioning functional model are inevitably influenced. In comparison, the closed-form Newton method can converge to the global optimal solution with better convergence and higher efficiency regardless of whether the initial iteration value is reasonable or not, especially with ill-conditioned positioning configuration. Furthermore, considering the high order partial derivatives, the closed-form Newton method can improve the accuracy of location estimation results compared to the Gauss-Newton method with increasing the computational cost a little. Therefore, in the application of indoor positioning, when the positioning configuration is ill conditioned and a good initial value cannot be obtained, we can choose to use the closed-form Newton method. Meanwhile, we generally try to avoid the ill-posed problems on the deployment of positioning system.

\section{Data Availability}

The data used to support the findings of this study are available from the corresponding author upon request.

\section{Conflicts of Interest}

The authors declare no conflicts of interest.

\section{Acknowledgments}

This work was supported by the National Natural Science Foundation of China (41874029) and the Introduction \& Training Program of Young Creative Talents of Shandong Province. 


\section{References}

[1] L. Wang, Z. Li, J. Zhao et al., "Smart device-supported BDS/ GNSS real-time kinematic positioning for sub-meter-level accuracy in urban location-based services," Sensors, vol. 16, no. 12, p. 2201, 2016.

[2] X. Li and S. Yang, "The Indoor Real-Time 3D Localization Algorithm Using UWB," in Proceedings of the 2015 International Conference on Advanced Mechatronic Systems, pp. 337-342, IEEE, Beijing, China, August 2015.

[3] A. Ferreira, D. Fernandes, A. Catarino et al., "Performance analysis of ToA-based positioning algorithms for static and dynamic targets with low ranging measurements," Sensors, vol. 17, no. 8, p. 1915, 2017.

[4] A. Alarifi, A. M. Al-Salman, M. Alsaleh et al., "Ultra wideband indoor positioning technologies: analysis and recent advances," Sensors, vol. 16, no. 5, p. 707, 2016.

[5] P. J. G. Teunissen, "Nonlinear inversion of geodetic and geophysical data: diagnosing nonlinearity," in Developments In Four-Dimensional Geodesy, pp. 241-264, Springer, Berlin, Heidelberg, 1990.

[6] R. O. Schmidt, "A new approach to geometry of range difference location," IEEE Transactions on Aerospace and Electronic Systems, vol. AES-8, no. 6, pp. 821-835, 1972.

[7] J. L. Awange and E. W. Grafarend, "Algebraic solution of gps pseudo-ranging equations,” GPS Solutions, vol. 5, no. 4, pp. 20-32, 2002.

[8] S. Q. Xue and Y. X. Yang, "Gauss-Jacobi combinatorial adjustment and its modification," Survey Review, vol. 46, no. 337, pp. 298-304, 2014.

[9] J. L. Awange and E. W. Grafarend, "Groebner-basis solution of the three-dimensional resection problem (P4P)," Journal of Geodesy, vol. 77, no. 5-6, pp. 327-337, 2003.

[10] J. L. Awange, E. W. Grafarend, Y. Fukuda, and S. Takemoto, "Direct polynomial approach to nonlinear distance (ranging) problems," Earth, Planets and Space, vol. 55, no. 5, pp. 231-241, 2003.

[11] J. L. Awange and Y. Fukuda, "Resultant optimization of the three-dimensional intersection problem," Survey Review, vol. 39, no. 304, pp. 100-108, 2007.

[12] N. Sirola, "A versatile algorithm for local positioning in closed form," in Proceedings of the 8th European Navigation Conference GNSS, Rotterdam Netherland, May 2004.

[13] B. T. Fang, "Simple solutions for hyperbolic and related position fixes," IEEE Transactions on Aerospace and Electronic Systems, vol. 26, no. 5, pp. 748-753, 1990.

[14] Y. T. Chan and K. C. Ho, "A simple and efficient estimator for hyperbolic location," IEEE Transactions on Signal Processing, vol. 42, no. 8, pp. 1905-1915, 1994.

[15] K. W. Cheung, H. C. So, W.-K. Ma, and Y. T. Chan, "Least squares algorithms for time-of-arrival-based mobile location," IEEE Transactions on Signal Processing, vol. 52, no. 4, pp. 1121-1128, 2004.

[16] W. Foy, "Position-location solutions by taylor-series estimation," IEEE Transactions on Aerospace and Electronic Systems, vol. 12, no. 2, pp. 187-194, 1976.

[17] P. Xu, "A hybrid global optimization method: the multi-dimensional case," Journal of Computational and Applied Mathematics, vol. 155, no. 2, pp. 423-446, 2003.

[18] P. J. G. Teunissen, "Nonlinear least squares," Manuscripta Geodaetica, vol. 15, no. 3, pp. 137-150, 1990.

[19] J. Yan, C. C. J. M. Tiberius, G. Bellusci, and G. J. M. Janssen, "Feasibility of gauss-newton method for indoor positioning," in Proceedings of the 2008 IEEE/ION Position, Location and
Navigation Symposium, pp. 660-670, Monterey, CA, USA, May 2008.

[20] A. Y. Ng, "Feature selection, 11 vs. 12 regularization, and rotational invariance," in Proceedings of the twenty-first international conference on Machine learning, Alberta Canada, July 2004.

[21] K. Madsen, H. B. Nielsen, and O. Tingleff, Methods for Nonlinear Least Squares Problems, Technical University of Denmark, Kongens Lyngby, Denmark, 2004.

[22] N. Sirola, "Closed-form algorithms in mobile positioning: myths and misconceptions," in Proceedings of the 2010 7th Workshop on Positioning, Navigation and Communication, pp. 38-44, Dresden, Germany, March 2010.

[23] S. Xue, Y. Yang, and Y. Dang, "Barycenter method for solving distance equations," Survey Review, vol. 48, no. 348, pp. 188-194, 2016.

[24] K. Levenberg, "A method for the solution of certain nonlinear problems in least squares," Quarterly of Applied Mathematics, vol. 2, no. 2, pp. 164-168, 1944.

[25] D. W. Marquardt, "An algorithm for least-squares estimation of nonlinear parameters," Journal of the Society for Industrial and Applied Mathematics, vol. 11, no. 2, pp. 431-441, 1963.

[26] Y. Dang and S. Xue, "A new Newton-type iterative formula for over-determined distance equations," in Earth on the Edge: Science for a Sustainable Planet, pp. 607-613, Springer, Berlin, Germany, 2014.

[27] S. Xue, Y. Yang, and Y. Dang, "A closed-form of Newton method for solving over-determined pseudo-distance equations," Journal of Geodesy, vol. 88, no. 5, pp. 441-448, 2014.

[28] K. Yu and I. Oppermann, "UWB positioning for wireless embedded networks," in Proceedings of the 2004 IEEE Radio and Wireless Conference (IEEE Cat. No.04TH8746), pp. 459462, Atlanta, GA, USA, September 2004.

[29] M. J. D. Powell, "How bad are the bfgs and dfp methods when the objective function is quadratic?" Mathematical Programming, vol. 34, no. 1, pp. 34-47, 1986.

[30] J. Sidorenkoab, V. Schatza, N. Scherer-Negenborna et al., "Decawave UWB clock drift correction and powerself-calibration," Sensors, vol. 19, no. 3, p. 2942, 2019.

[31] G. Shen, R. Zetik, and R. S. Thoma, "Performance comparison of toa and tdoa based location estimation algorithms in los environment," in Proceedings of the 2008 5th Workshop on Positioning, Navigation and Communication, pp. 71-78, Hannover, Germany, March 2008.

[32] J. Yan, C. C. J. M. Tiberius, P. J. G. Teunissen, G. Bellusci et al., "A framework for low complexity least-squares localization with high accuracy," IEEE Transactions on Signal Processing, vol. 58, no. 9, pp. 4836-4847, 2010. 